Ludwig M. Eichinger

\title{
Deutsch im Sprachvergleich. Grammatische Kontraste und Konvergenzen
}

Er war so witzig, daß jedes Ding ihm gut genug war zu einem Mittelbegriff jedes Paar andere Dinge mit einander zu vergleichen. ( J.Ch. Lichtenberg, Sudelbücher B1 101)

\section{Vergleichen}

\subsection{Im Allgemeinen}

Das Vergleichen gehört zur Wissenschaft - so könnte man Thomas von Aquins scholastischen Kernspruch scientia non est de singularibus auch verstehen. ${ }^{1}$

Wenn das so ist, vermag es nicht zu verwundern, dass der Vergleich sprachlicher Verhältnisse in der Geschichte der Jahrestagungen des Instituts für Deutsche Sprache schon mehrfach zum Thema gewählt wurde. Es versteht sich von selbst, dass mit einem vergleichbaren Thema im Wechsel der Zeiten nicht die gleichen und schon gar nicht dieselben Interessen verbunden sind. Gerade deswegen aber lohnt es sich, einen Blick auf die früheren Tagungen zu werfen, die sich dem Thema des Sprachvergleichs in der einen oder anderen Weise gewidmet haben. Diese Rückschau wirft ein bezeichnendes Licht darauf, welche Wege die Diskussion um das Vergleichen von Sprachen und von sprachlichen Verhältnissen in den Jahrzehnten seit der Gründung des Instituts genommen hat.

\subsection{Die Zwei Seiten: Kontrast und Konvergenz}

Welches der Blickwinkel ist, der die Beschäftigung mit diesem Thema prägt, wird schon im Untertitel des Tagungsthemas deutlich. Um grammatische Kontraste und Konvergenzen sollte es also im Jahr 2011 gehen. Dass der Vergleich grammatischer Strukturen im Fokus stehen soll, davon soll etwas später noch die Rede sein. Charakteristischer ist vielleicht, dass bei der diesjährigen Tagung dezidiert auf zwei Verhältnisse und Entwicklungen gleichzeitig geblickt werden soll: auf die - augenfälligen - Verschiedenheiten ohnehin, aber eben gleichwertig auch darauf, was ohnehin schon nicht nur vergleichbar, sondern ähnlich ist, und vielleicht sogar Entwicklungen zeigt,

Zum Ort und historischen Stellenwert dieser Aussage siehe Jacobi (2008, S. 75 f.). 
die aufeinander zu gehen. Die Aufmerksamkeit gilt der Diversifikation und dem Zusammengleichen zwischen Sprachen gleichermaßen. Das reflektiert eine Herangehensweise, die notwendig mit den Zielen der neueren grammatischen Arbeit am IDS verbunden ist. Wenn man sich an eine Grammatik des Deutschen im europäischen Vergleich heranwagt, hat man eine komplizierte Balance zu leisten. Man hat eine Ebene des Vergleichs zu finden, die zumindest im Hinblick auf das, was wir aus allgemein typologischen und spezieller eurotypologischen Studien wissen, einerseits zu einer grammatischen Beschreibung des Deutschen passt, also Kategorien betrifft, die sich in einer grammatischen Beschreibung des Deutschen niederschlägt. ${ }^{2}$ Es ist die andere Seite einer ausgewogenen vergleichenden Beschreibung, dass die Ebene auch geeignet sein muss, die Verhältnisse in den Vergleichssprachen aus ihrem eigenen Blickwinkel zu beschreiben. Damit hängt zusammen, dass letztlich hier wie dort die spezifischen Traditionen grammatischer Beschreibung mit zu bedenken sind. Es ist die Ebene von funktionalen Domänen - wie etwa Determination ${ }^{3}$-, die als Basis eines solcherart ausgeglichenen Vergleichs genommen werden. Vor diesem Stand der Überlegungen am Institut für Deutsche Sprache ausgehend wurde die Tagung des Jahres 2011 geplant.

\section{Verschiedene Konzepte}

\subsection{Kontrastive Grammatik}

Dass genau dieser Kontext der Vergleichs-Diskussion den Unterschied ausmacht, wenn man in der Geschichte der Tagungen zurückblickt, ist ebenso augenfällig wie wenig überraschend. Wenn sich so eine der frühesten Jahrestagungen, die damals noch Jahressitzung des Wissenschaftlichen Rates des Instituts hieß, der „Kontrastiven Grammatik“ widmete, so setzt auch hier der Kontext der Zeit ihren Akzent. Auch hier gibt schon die Formulierung des Titels einen leitenden Hinweis. Die damalige Diskussion einer solchen vergleichenden Grammatikforschung fand im Umfeld einer seit den späten 1950er Jahren entstandenen kontrastiven Linguistik statt, die sich als eine angewandte Subdisziplin präsentierte, die im Fremdsprachenunterricht ihre Anwendung fand. Im Kern handelt es sich um den Spezialfall einer

\footnotetext{
2 Die in einer entsprechenden Weite gewählt werden müssen, um sowohl die internen Zusammenhänge wie den Weg zu nachbarsprachlichen Optionen nicht zu verstellen; vgl. den Einbezug der Wortbildung.

3 Dieses Beispiel ist gewählt, weil es für die Beschreibung des Deutschen dem Bereich der Nominalgrammatik zugehört, die im Rahmen des Projektes GDE derzeit bearbeitet wird. Entsprechend sind auch im Projekt „Eurogr@mm“ Ergänzungen aus vergleichender Perspektive entstanden, die den nominalen Bereich betreffen; vgl. http://hypermedia.ids-mannheim. $\mathrm{de} /$ pls/public/gruwi.ansicht?v_typ=0.
} 
Fehlerlinguistik, die nach strukturellen Unterschieden (zumeist von zwei Sprachen) sucht, die als potenzielle Fehlerquellen angesehen werden. Man hatte offenbar schon gleichzeitig den Eindruck, dass ihr Vorgehen im Wesentlichen auf nicht hinreichend überprüften Plausibilitätsannahmen beruhte. ${ }^{4}$ Ohne dass durch die verschiedenen Beiträge hindurch ein gänzlich gefestigter Gebrauch dieses Terminus erkennbar wäre, geht es offenbar darum, sich von einer nur praktisch ausgerichteten, an fehlerträchtigen Kontrasten interessierten kontrastiven Untersuchung abzusetzen. Das Ziel mag dabei Verschiedenes sein, im - theoretischen - Kern geht es darum, eine angemessene Ebene des Vergleichs zu finden. Es ist wenig überraschend, dass sich an der Bedeutung dieser kritischen Stelle der Vergleichsdiskussion, wie wir gesehen haben, seither nichts Grundlegendes geändert hat. Geändert haben sich allerdings die Bezugskategorien des Vergleichs. Ist es in der heutigen Diskussion in dem grammatischen Projekt des IDS, der „Grammatik im europäischen Vergleich“ (GDE) eine funktionale Kategorie, die der funktionalen Domäne, die eine typologisch angemessene neutralmöglichste Sicht auf die Verhältnisse ermöglichen soll, ging es damals um die Bestimmung und Festlegung der relevanten Ebene im Rahmen der vorherrschenden strukturalistischen Diskussion. Ganz offenkundig war die Intention nicht, sich von einer wie auch immer gearteten Praxis abzusetzen, sondern ihr einen wissenschaftlichen Grund zu geben. Im Leitbeitrag zu dieser Tagung, der von Eugenio Coseriu eingebracht wurde, heißt es dazu:

Die Unterschiede, welche die Ebene des Sprachtypus betreffen, obschon wissenschaftlich hochinteressant, sind freilich in praktischer Hinsicht nicht von unmittelbarem Belang. Dagegen ist die Unterscheidung zwischen Sprachsystem und Sprachnorm [...] gerade auch für die praktische Erlernung der Sprachen wichtig und unentbehrlich. (Coseriu 1969, S. 27)

Gerade im Hinblick auf eine solcherart an der Sprachnorm orientierte Untersuchung, die Inhalts- und Funktionseinheiten über die Sprachen hin verfolgt, heißt es dann im Vorwort des Tagungsbandes:

Die komparative oder konfrontative Sprachbeschreibung kann, um eine Formulierung von Coseriu zu gebrauchen, auch für das Deutsche, nach dieser Tagung als begründet gelten'. (Moser et al., S. 7)

Auch die damalige Diskussion stand im Zusammenhang mit entsprechenden Arbeiten am Institut. In den 1970er Jahren begannen die Arbeiten an kontrastiven und vergleichenden Grammatiken, die auf jeden Fall einen systematischeren Gesamtvergleich anstrebten als er in dem geschilderten Kontext vorgesehen war. Tatsächlich sind dann Ende der 1970er, Anfang der 1980er Jahre zwei große vergleichende Grammatiken erschienen, die in ihrer

Zur damaligen kontrastiven Linguistik vgl. Nickel (Hg.) (1972) und Nickel (1980); zur kritischen Einschätzung z.B. Christian Lehmanns Zusammenfassung unter www.christianlehmann. eu/ling/elements/index.html?http://www.christianlehmann.eu/ling/elements/kontr_ling.html. 
Verschiedenheit die Bandbreite dessen zeigen, was man sich unter einer an Vollständigkeit orientierten vergleichenden Grammatik vorstellte. Will die mit dem Spanischen vergleichende Grammatik von Cartegena/Gauger (1989) in einer eher an der traditionellen Grammatikschreibung orientierten Gegenüberstellung wesentliche Unterschiede und Charakteristika hervorheben, bringt Jean-Marie Zembs (1978 und 1984) deutsch-französische Grammatik eine Darstellung, in der die Verhältnisse in der deutschen Sprache auf Französisch und die in der französischen Sprache auf Deutsch ausgebreitet werden, jeweils aus ihrer eigenen Art und in einer eigenen Art von Grammatik. Daneben wird in dieser Zeit die Basis für eine Reihe von kontrastiven Grammatiken gelegt, die von Ulrich Engel initiiert wurden, und die ihren Ausgang auch von der valenzgrammatischen Beschreibung nimmt, die Ulrich Engel in seinen Grammatiken vorgeschlagen hat. ${ }^{5}$ Hier ermöglicht die quasi-axiomatische Setzung dieses Modells den Vergleich, der sich allerdings auch wandelt, wenn man die früheren Exemplare zum Beispiel mit der deutsch-polnischen kontrastiven Grammatik vergleicht, in die auf jeden Fall auch mehr von der eigenständigen polnischen Sicht und entsprechenden Beschreibungstraditionen eingegangen ist. Es gibt sicherlich verschiedene Gründe, warum dieser Typ von Arbeiten dann irgendwann am IDS nicht mehr fortgeführt worden ist; einer dürfte auf jeden Fall gewesen sein, dass sich in der Durchführung zeigte, dass offenkundig keine Vorstellung davon existierte, nach welchem Muster vergleichende Grammatiken gestaltet werden sollten, sofern sie nicht konfrontativ im Sinne didaktischer Nutzbarkeit waren.

Geht es bei der ersten Tagung wie in diesen Forschungen zuvorderst darum, die Eigenheiten der eigenen Sprache in der Gegenüberstellung mit einer anderen zu erhellen, wie Eduard Beneš im Kontext der Tagung explizit feststellt, so hat sich bei der nächsten Gelegenheit der Weg der Annäherung an das Deutsche umgekehrt.

\subsection{Typologie}

Das nächste Mal, das ist das Jahr 1995, als der programmatische Titel der Jahrestagung „Deutsch typologisch“ lautete. ${ }^{6}$ Damals jedenfalls ging es darum, an einer Reihe von Erscheinungen quer über die grammatischen Ebe-

\footnotetext{
$5 \quad$ Als die zuletzt erschienene dieser Grammatiken, die nicht aus IDS-Projekten hervorgingen, sei die deutsch-polnische Grammatik (Engel 1999) erwähnt, deren zweite Auflage in Vorbereitung ist. Weitere kontrastive Vorhaben führten nicht zu solch „handbuchartigen“ Ergebnissen, vgl. zum deutsch-japanischen Stickel (2001).

$6 \quad$ Vorbereitet wurde diese Tagung seinerzeit im Hause des IDS von Gisela Zifonun, die damals vermutlich in ähnlicher Weise in den Schlussarbeiten zu der 1997 erscheinenden großen IDSGrammatik (Zifonun/Hoffmann/Strecker 1997) steckte, wie sie das heute mit dem Nominalband der Grammatik des Deutschen im europäischen Vergleich tut, auf dessen Erscheinen in analoger Weise wir uns dann freuen.
} 
nen hin zu bestimmen, wo sich das Deutsche in anzunehmenden universalen Ordnungen positioniere. Und Ewald Lang als damaliger Mitorganisator hält als zentrale Leitlinien der Tagung fest:

1) Typologie und Universalien stehen im Konnex [...] 2) Die typologischen Merkmale bilden ein Raster grammatischer Optionen, [...] 3) Das so verstandene typologische Raster bildet einen geeigneten Rahmen, in den kontrastive Studien einzubetten sind. (Lang 1996, S. 8)

Und im Vorwort des damaligen Bandes wird er von Gerhard Stickel mit dem folgenden Bild zitiert:

Bei der typologischen Beschreibung des Deutschen gehe es darum, meinte Lang, eine Art Phantombild einer Sprache zu erstellen. So wie bei der kriminalistischen Arbeit ein Bild des Verdächtigen durch die Auswahl aus typischen Nasen, Haaransätzen, Augen, Kinnformen und anderen physiologischen Merkmalen schrittweise bis zur Erkennbarkeit rekonstruiert wird, sucht die typologische Beschreibung durch Analyse klassifizierungsgeeigneter Eigenschaften wie Satzstellung, Wortmorphologie [usw.] das charakteristische Profil dieser Sprache zu ermitteln. (Stickel 1996, S. 2)

Man kann auch in diesem Fall versuchen, die thematische Wendung mit den Diskussionen um die Forschungsausrichtung des Hauses zu verbinden. Die große IDS-Grammatik ist zu diesem Zeitpunkt faktisch abgeschlossen. Sie hat in grundsätzlicher Hinsicht andere Punkte fokussiert. Sie hat einerseits eine Konsistenz und methodische Verlässlichkeit erreicht, eine Faktenfülle geordnet und das Konzept einer Grammatik der Äußerung in einer Weise realisiert, die ihren Rang als ein Handbuch, das linguistische Grundlagenforschung mit einer flächendeckenden Beschreibung der Verhältnisse des Deutschen begründet. Eigentlich waren für das Haus aufgrund der vertieften Kenntnis der grammatischen Verhältnisse des Deutschen und der gesammelten Erkenntnisse zur Beschreibung dieser Verhältnisse die Voraussetzungen dazu gegeben, sich in verlässlicher Weise - und mit begründeter Hoffnung auf eine Ausbeute für die eigene weitere Forschung - mit der typologischen Verortung der verschiedensten Fakten der grammatischen Erscheinungen des Deutschen zu beschäftigen - und sich so auch in die aktuelle linguistische Diskussion einzubringen und einzumischen. ${ }^{7}$ Wenn man nun nach weit über einem Jahrzehnt weiterer Forschung auf die Beiträge zurückblickt, die auf dieser Tagung präsentiert wurden, so sieht man, dass es nicht zuletzt der Erfolg der damaligen großen eurotypologischen Forschungsprojekte war, ${ }^{8}$ die Forscher verschiedenster theoretischer und methodischer Provenienz dazu brachte, sich mit dieser Frage zu beschäftigen.

Die eine hohe allgemeine Sichtbarkeit erreicht hatte; vgl. exemplarisch Haspelmath et al. (2005); siehe auch www.eva.mpg.de/lingua/tools-at-lingboard/questionnaire/eurotyp_description.php.

Vgl. die seit 1998 erschienenen Bände der Eurotyp-Reihe bei de Gruyter. Das ist vielleicht der rechte Ort, um auf das bemerkenswerte Faktum hinzuweisen, dass auch auf dieser 
Bei dieser Lage ist es klar, dass dann doch in den einzelnen Beiträgen ein unterschiedliches Bild vom Vergleichen aufscheint. Wie immer es im Einzelnen allerdings ausgestaltet wird: Wenn man es im Lichte der Entwicklung sieht, die wir hier anzudeuten versuchen, geht es tatsächlich darum, wie es in einem der allgemein grundlegenden Beiträge dieses Bandes, dem von Ekkehard König, heißt, ,kontrastive Grammatik als Grenzfall der Typologie“ (König 1996, S. 31) zu verstehen. Das hat erkennbar zwei Folgen. Zum einen, und das ist vielleicht auch für die praktische Arbeit am IDS folgenreich geworden, geht es nicht mehr darum, Eigentümlichkeiten von zwei Sprachen gegeneinander zu halten, sondern das Profil, der Sprachtyp erhellt sich aus dem Ort des Deutschen im Rahmen der Optionen von Sprachen überhaupt. Praktisch heißt das zumindest, in einer vergleichenden Sicht auf eine größere Zahl von Sprachen. Ob man dazu eine unter irgendeinem Aspekt gewählte Gruppe näherstehender Sprachen wählt oder die Differenz auszuspannen bemüht ist, hängt vielleicht davon ab, was das Ziel der jeweiligen Forschung ist. Zum anderen ist das Bemühen erkennbar, diese typologisch gestützte Einordnung des Deutschen nicht auf eine Ebene der sprachlichen Beschreibung zu beziehen, sondern auch in dieser Hinsicht ein aufgefächertes Profil der deutschen Sprache zu liefern. Man kann da einerseits an typologisch zweifellos Bewährtem ansetzen - etwa den typologisch relevanten Serialisierungsmustern -, man kann herausgehobenen Phänomenen nachgehen, bei denen die Realisierung einer grammatischen Kategorie mit einer hohen funktionalen Eindeutigkeit - wie etwa Negation auf ihre typologische Einbettung untersucht wird. Man kann aber auch grundsätzliche Annahmen - wie etwa Ikonizität - zur Basis des Vergleiches wählen. Letztlich ist das natürlicherweise der Anlass, die Annahmen einer Theorie mit dem Anspruch auf die Beschreibung universaler Strukturen mit typologischen Verbindungen und Untergliederungen zu kontrastieren. Diese durch die typologischen Forschungen „provozierten“ Vergleiche verschiedener Art werfen ein erhellendes Licht auf verschiedene Weisen linguistischen Arbeitens. So nimmt es nicht Wunder, dass die damals in Mannheim geführten Diskussionen in bemerkenswertem Ausmaße in der fachlichen Diskussion rezipiert wurden.

Tagung Ekkehard König (vgl. König 1996), einen der eröffnenden Vorträge mit dem Titel „Kontrastive Grammatik und Typologie“" gehalten hat, bei dem es unter anderem darum geht, was es in diesem Kontext bedeutet, dass das Deutsche eine germanische Sprache ist. Im vorliegenden Band finden wir ihn ja ebenfalls mit dem einführenden Beitrag ,Zur Standortbestimmung der Kontrastiven Linguistik innerhalb der vergleichenden Sprachwissenschaft“". Dass er auch schon auf der ersten erwähnten Tagung referiert hat (König/Nickel 1970), rundet das Bild ab. 


\subsection{Typologisch basierter Vergleich}

Seither ist wiederum mancherlei geschehen. Die eurotypologische Forschung hat uns einen neuen Blick gelehrt, die Zusammenhänge in unserem, wenn man so will, sprachlichen Nahraum, sind so viel präziser beschrieben worden, im Kontext damit ist klarer geworden, dass auch das gemeinsame sprachliche Leben über Jahrhunderte hin in der Nachbarschaft vieler Sprachen all diese Sprachen nicht unbeeindruckt lässt, was in den Blick kommt, wenn man ihre Eigenheiten herauszubringen versucht. Und es hat sich herausgestellt, dass das Deutsche in einem „normalen“ europäischen Kontext oft als „Mischtyp“ zu charakterisieren wäre 9 also in mancherlei Hinsicht in der Mitte steht. Erfreulicherweise haben wir bei der Jahrestagung 2002 von Cathrine Fabricius-Hansen (2003) gelernt, dass manches, das wir als häufig ironisierte Eigenschaften des Deutschen ansehen, seine Rechtfertigung aus den Anforderungen an eine „reife Sprache“ zieht. Das alles ist geschehen, als sich das Institut aufmachte, in ständigem Vergleich mit einer Reihe europäischer Nachbar- und Lernsprachen einer Grammatik im europäischen Vergleich nachzugehen: ${ }^{10}$ Was das heißt, und welche methodisch-theoretischen Fragen damit verbunden sind, ${ }^{11}$ will und muss ich hier nicht mehr zu erläutern versuchen, ${ }^{12}$ dafür sind die hier abgedruckten Beiträge der Tagung da - und die diesem Beitrag folgende Einführung in das Tagungsthema durch Lutz Gunkel und Gisela Zifonun. Von der Vielseitigkeit der Vergleiche ${ }^{13}$ und der Fundierung der eigenen Forschungen in einem typologisch basierten Konzept ${ }^{14}$ zeugen eine Vielzahl von neueren Publikationen aus der Projektgruppe und ihrem Umfeld. Zudem hat diese Art des Vergleichens weiter dazu geführt, dass auch die stärker anwendungsorientierte Forschung auf die Basis eines vielfältigen und multidirekationalen Vergleichs gestellt werden konnte.Im Projekt „Eurogr@mm“konnte eine entsprechende plurinationale Arbeitsgruppe zusammengestellt werden, die den „fremden Blick“ auf das Deutsche systematisiert. ${ }^{15}$

\footnotetext{
$9 \quad$ Vgl. dazu z.B. Askedal (1996).

10 Eine Reihe von Vorstudien und Präsentationen erster Ergebnisse zeigt, von welcher Art die Ergebnisse sind, die hier zu erwarten sind; vgl. z.B. die vier Publikationen zu Pronomina (Zifonun 2001 bis Zifonun 2007).

11 Dazu hat sich die Projektleiterin, was die Grammatik des Deutschen im europäischen Vergleich angeht, z.B. in Zifonun (2008) geäußert.

12 Vgl. aber die in Eichinger (2008) angestellten Überlegungen.

13 Beispiele dafür sind etwa Gunkel/Schlotthauer (2009), für den Bereich „entfernterer“ Sprachen, oder Gunkel/Zifonun (2011) für den Bereich der klassischen Schulsprachnachbarn.

14 Auch hier sollen einige Hinweise genügen, so werden Fragen der Flexionsmorphologie ebenso in vielfältiger Weise diskutiert (vgl. z.B. Wiese 2009 und 2011 oder den Sammelband Müller/ Gunkel/Zifonun (Hg.) 2004), wie etwa Fragen der Modifikation (vgl. Gunkel/Rijkhoff (Hg.) 2009).

15 Die Ergebnisse gehen als das Modul „Progr@mm kontrastiv“ in den Rahmen des Projekts Progr@mm ein (siehe Schwinn/Augustin 2007-2009). Zudem erscheint demnächst eine erste zusammenfassende Publikation (Augustin/Fabricius-Hansen im Druck).
} 
Es gibt und gab also genügend Anlass, wieder einmal über das Vergleichen - seine Voraussetzungen und seine Ergebnisse - nachzudenken. Die Entwicklung eines Konzepts und eines Programms für eine Tagung, die dem nachkommen sollte, lag in den Händen von Gisela Zifonun und Lutz Gunkel aus dem Haus sowie des Kollegen Jörg Meibauer und von Frau Kollegin Martine Dalmas. Ihnen allen sei gedankt für die Vorbereitung dieser Jahrestagung. Ein besonders herzlicher Dank geht an alle Referenten, die die Tagung ja ausmachen und an alle, die für ihren glatten Ablauf sorgen.

\section{Schluss}

Manchmal spreche ich an dieser Stelle über das Wetter, aber es gibt Gelegenheiten, um einen Spruch von Bert Brecht zu paraphrasieren, wo das Gespräch über das Wetter nicht das passendste ist. Das scheint mir in diesem Jahr der Fall zu sein. So grüße ich am Ende meines Vorworts unsere japanischen Kollegen, die, die hier waren, und die, die nicht kommen konnten, deren Sorgen wir teilen, und für deren Lage wir nur das Beste wünschen können. ${ }^{16}$

\section{Literatur}

Askedal, John Ole (1996): Überlegungen zum Deutschen als sprachtypologischem „Mischtyp“. In: Lang, Ewald/Zifonun, Gisela (Hg.): Deutsch - typologisch. (= Jahrbuch des Instituts für Deutsche Sprache 1995). Berlin, S. 369-383.

Augustin, Hagen/Fabricius-Hansen, Cathrine (Hg.) (i.Dr.): Flexionsmorphologie des Deutschen im Kontrast. Tübingen.

Cartagena, Nelson/Gauger, Hans-Martin (1989): Vergleichende Grammatik SpanischDeutsch. 2 Bde. (= Vergleichende Grammatiken 2). Mannheim/Wien/Zürich.

Coseriu, Eugenio (1969): Über Leistung und Grenzen der kontrastiven Grammatik. In: Wissenschaftlicher Rat des IDS (Hg.), S. 9-30.

Eichinger, Ludwig M. (2008): Deutsch als europäische Sprache. In: Eichinger, Ludwig M./Plewnia, Albrecht (Hg.): Das Deutsche und seine Nachbarn. Über Identitäten und Mehrsprachigkeit. (= Studien zur Deutschen Sprache 46). Tübingen, S. 13-30.

Engel, Ulrich (1999): Deutsch-polnische kontrastive Grammatik. Unter Mitarbeit von Rytel-Kuc, D., Cirko, L., Debski, A. u.a. 2 Bde. Heidelberg.

$16 \quad$ Die Jahrestagung 2011 fand unmittelbar nach dem Erdbeben und dem folgenden Tsunami im Nordosten Japans sowie dem Atomunglück von Fukushima statt. Ich dachte, man sollte diesen Bezug nicht tilgen: Das erklärt jedenfalls und entschuldigt vielleicht die Beibehaltung der Anredeform an dieser Stelle. 
Fabricius-Hansen, Cathrine (2003): Deutsch - eine ,reife' Sprache. Ein Plädoyer für die Komplexität. In: Stickel, Gerhard (Hg.): Deutsch von außen. (= Jahrbuch des Instituts für Deutsche Sprache 2002). Berlin/New York, S. 99-112.

Gunkel, Lutz/Schlotthauer, Susan (2009): Attribution in Basque, Finnish, Hungarian and Turkish: morphology vs. syntax. In: Saxena, Anju/Viberg, Åke (Hg.): Multilingualism. (= Studia Linguistica Upsaliensia 8). Uppsala, S. 51-64.

Gunkel, Lutz/Zifonun, Gisela (2011): Klassifikatorische Modifikation im Deutschen und Französischen. In: Lavric, Eva/Pöckl, Wolfgang/Schallhart, Florian (Hg.): Comparatio delectat. (= InnTrans 4). Frankfurt a.M. u.a., S. 549-562.

Gunkel, Lutz/Rijkhoff, Jan (Hg.) (2010): Modifikation im Deutschen: Kontrastive Untersuchungen zur Nominalphrase. Themenheft der Zeitschrift Deutsche Sprache (Heft 2/2010).

Haspelmath, Martin et al. (2005): World atlas of language structures. Oxford.

Jacobi, Klaus (2008): „Actus circa singularia sind“ - „scientia non est de singularibus“. Thomas von Aquins Konzeption einer praktischen Wissenschaft. In: Lutz-Bachmann, Matthias/Fidora, Alexander (Hg.): Handlung und Wissenschaft. Die Epistemologie der praktischen Wissenschaften im 13. Und 14. Jahrhundert. Berlin, S. $75-88$.

König, Ekkehard (1996): Kontrastive Grammatik und Typologie. In: Lang/Zifonun (Hg.), S. 31-54.

König, Ekkehard/Nickel, Gerhard (1970): Transformationelle Restriktionen in der Verbalsyntax des Englischen und Deutschen. In: Moser, Hugo (Hg.): Probleme der kontrastiven Sprachwissenschaft. (= Jahrbuch des Instituts für Deutsche Sprache 1969). Düsseldorf, S. 70-81.

Lang, Ewald (1996): Das Deutsche im typologischen Spektrum. Einführung in den Band. In: Lang/Zifonun (Hg.), S. 7-15.

Lang, Ewald/Zifonun, Gisela (Hg.) (1996): Deutsch typologisch. (= Jahrbuch des Instituts für Deutsche Sprache 1995). Berlin/New York.

Moser, Hugo et al. (1969): Geleitwort. In: Wissenschaftlicher Rat des IDS (Hg.), S. 7.

Müller, Gereon/Gunkel, Lutz/Zifonun, Gisela (Hg.) (2004): Explorations in nominal inflection. (= Interface Explorations 10). Berlin.

Nickel, Gerhard (Hg.) (1972): Fehlerkunde: Beiträge zur Fehleranalyse, Fehlerbewertung und Fehlertherapie. Berlin.

Nickel, Gerhard (1980): Kontrastive Linguistik. In: Althaus, Peter/Henne, Helmut/Wiegand, Herbert E. (Hg.): Lexikon der Germanistischen Linguistik. 2., vollst. neu bearb. u. erw. Aufl. Tübingen, S. 633-636.

Schwinn, Horst/Augustin, Hagen (2007-2009): ProGr@mm - Propädeutische Grammatik des Instituts für Deutsche Sprache. Mannheim. Internet: www.ids-mannheim. de/progr@mm/ (Stand: 18.11.2011).

Stickel, Gerhard (1996): Eröffnung der Jahrestagung 1995. In: Lang/Zifonun (Hg.), S. 1-3. 
Stickel, Gerhard (Hg.) (2001): Deutsch-japanische Kontraste. Vorstudien zu einer kontrastiven Grammatik. (= Forschungsberichte des Instituts für Deutsche Sprache 29). Mannheim.

Wiese, Bernd (2009): Variation in der Flexionsmorphologie: Starke und schwache Adjektivflexion nach Pronominaladjektiven. In: Konopka, Marek/Strecker, Bruno (Hg.): Deutsche Grammatik - Regeln, Normen, Sprachgebrauch. (= Jahrbuch des Instituts für Deutsche Sprache 2008). Berlin/New York, S. 166-194.

Wiese, Bernd (2011): Optimal specifications: on case marking in Polish. In: Nolda, Andreas/Teuber, Oliver (Hg.): Syntax and morphology multi dimensional. (= Interface Explorations 24). Berlin/New York, S. 101-127.

Wissenschaftlicher Rat des IDS (Hg.) (1969): Jahrbuch 1969. Probleme der kontrastiven Grammatik. (= Sprache der Gegenwart 8). Düsseldorf.

Zifonun, Gisela (2001): Grammatik des Deutschen im europäischen Vergleich. Das Pronomen. Teil I: Überblick und Personalpronomen. (= amades 4/01). Mannheim.

Zifonun, Gisela (2007): Zur Grammatik des Deutschen im europäischen Vergleich. Das Pronomen. Teil IV: Indefinita im weiteren Sinne. (= amades 4/06). Mannheim.

Zifonun, Gisela (2008): Kontrastive Grammatik und Typologie, am Beispiel des Vergleichs zwischen dem Deutschen und dem Rumänischen. In: Stănescu, Speranța/ Engel, Ulrich (Hg.): Sprachvergleich - Kulturvergleich: Quo vadis, KGdr? München, S. 74-90.

Zifonun, Gisela/Hoffmann, Ludger/Strecker, Bruno (1997): Grammatik der deutschen Sprache. 3 Bde. (= Schriften des Instituts für Deutsche Sprache 7). Berlin/New York.

Zemb, Jean-Marie (1978 und 1984): Vergleichende Grammatik Französisch-Deutsch. Comparaison de deux systèmes. [Mit Beiträgen einer Vielzahl von Autoren]. (= Vergleichende Grammatiken Bd. 1.1 und 1.2). Mannheim/Wien/Zürich. 